\title{
The Schur Multiplier of Pairs of Groups of Order $p^{2} q$
}

\author{
S. Rashid, ${ }^{1}$ A. A. Nawi, ${ }^{2}$ N. M. Mohd Ali, ${ }^{2}$ and N. H. Sarmin ${ }^{2}$ \\ ${ }^{1}$ Department of Mathematics, Faculty of Science, Islamic Azad University, Firoozkooh Branch, Tehran 3319118651, Iran \\ ${ }^{2}$ Department of Mathematical Sciences, Faculty of Science, Universiti Teknologi Malaysia (UTM), 81310 Johor Bahru, Johor, Malaysia
}

Correspondence should be addressed to S. Rashid; samadrashid47@yahoo.com

Received 3 September 2014; Revised 5 November 2014; Accepted 5 November 2014; Published 25 November 2014

Academic Editor: Kishin Sadarangani

Copyright (c) 2014 S. Rashid et al. This is an open access article distributed under the Creative Commons Attribution License, which permits unrestricted use, distribution, and reproduction in any medium, provided the original work is properly cited.

Let $(G, N)$ be a pair of groups where $G$ is a group and $N$ is a normal subgroup of $G$. Then the Schur multiplier of pairs of groups $(G, N)$ is a functorial abelian group $M(G, N)$. In this paper, $M(G, N)$ for groups of order $p^{2} q$ where $p$ and $q$ are prime numbers are determined.

\section{Introduction}

The Schur multiplier was introduced by Schur [1] in 1904. The Schur multiplier of a group $G, M(G)$, is isomorphic to $R \cap[F, F] /[R, F]$ in which $G$ is a group with a free presentation $1 \rightarrow R \rightarrow F \rightarrow G \rightarrow 1$. He also computed $M(G)$ for many different kinds of groups: for example, the dihedral group, metacyclic group, alternating group, and quaternion group. All computations of $M(G)$ were then compiled by Karpilovsky [2] in a book entitled “The Schur Multiplier."

In 1998, Ellis [3] extended the notion of the Schur multiplier of a group to the Schur multiplier of a pair of group, $(G, N)$, where $N$ is a normal subgroup of $G$. The Schur multiplier of a pair of groups, $(G, N)$, is a functorial abelian group $M(G, N)$ whose principal feature is natural exact sequence

$$
\begin{gathered}
H_{3}(G) \stackrel{\eta}{\longrightarrow} H_{3}\left(\frac{G}{N}\right) \longrightarrow M(G, N) \longrightarrow M(G) \stackrel{\mu}{\longrightarrow} M\left(\frac{G}{N}\right) \\
\longrightarrow \frac{N}{[N, G]} \longrightarrow(G)^{a b} \stackrel{\alpha}{\longrightarrow}\left(\frac{G}{N}\right)^{a b} \longrightarrow 1,
\end{gathered}
$$

in which $H_{3}(-)$ denotes some finiteness-preserving functor from groups to abelian groups (to be precise, $H_{3}(-)$ is the third homology of a group with integer coefficients). The homomorphisms $\eta, \mu, \alpha$ are those due to the functorial of $H_{3}(-), M(-)$, and $(-)^{a b}$. Ellis [3] also stated that, for any pair $(G, N)$ of groups, $M(G, N) \cong \operatorname{ker}(N \wedge G \rightarrow G)$ where $N \wedge G$ is the exterior product of $N$ and $G$. The exterior product $N \wedge G$ is obtained from $N \otimes G$ by imposing the additional relation $n \otimes g=1$ for all $(n, g) \in N \wedge G$ and the image of a general element $n \otimes g$ in $N \wedge G$ is denoted by $n \wedge g$ for all $n \in N$ and $g \in G$.

The nonabelian tensor product, $G \otimes H$, was introduced by Brown and Loday [4] in 1987. $G \otimes H$ is the group generated by the symbols $g \otimes h$ subject to the relations

$$
\begin{aligned}
& g g^{\prime} \otimes h=\left({ }^{g} g^{\prime} \otimes{ }^{g} h\right)(g \otimes h), \\
& g \otimes h h^{\prime}=(g \otimes h)\left({ }^{h} g \otimes{ }^{h} h^{\prime}\right),
\end{aligned}
$$

for all $g, g^{\prime}$ in $G$ and $h, h^{\prime}$ in $H . G \otimes H$ is used in computing the Schur multiplier of the direct product of two groups, $M(G \times$ $H)$. Some computations of the nonabelian tensor product of cyclic group of $p$-power order have been done by Visscher [5] in 1998.

The nonabelian tensor square and Schur multiplier of groups of order $p^{2} q, p q^{2}$, and $p^{2} q r$ has been computed by Jafari et al. [6]. In this paper, the Schur multiplier of pairs of groups of order $p^{2} q$ where $p$ and $q$ are primes is determined.

In 2007, Moghaddam et al. [7] showed that $M(G, N) \cong$ $R \cap[S, F] /[R, F]$ if $S$ is a normal subgroup of $F$ such that $N \cong$ $S / R$. In 2012, Rashid et al. [8] determined the commutator subgroups of groups of order $8 q$. The Schur multiplier, nonabelian tensor square, and capability of groups of order $p^{2} q$ have been considered by Rashid et al. in [9], where $p$ 
and $q$ are distinct primes. In [10], they also computed the nonabelian tensor square and capability of groups of order $8 q$, where $q$ is an odd prime.

\section{Preliminaries}

This section includes some preliminary results that are used in proving our main theorems.

Definition 1 (see [2]). A normal subgroup $N$ of $G$ is called a normal Hall subgroup of $G$ if the order of $N$ is coprime to its index in $G$.

Definition 2 (see [2]). $M(N)^{T}$ is defined as the $T$-stable subgroup of $M(N)$; that is, $M(N)^{T}=\left\{f \in M(N) \mid \operatorname{Con}_{N}^{t}(f)=\right.$ $f$ for all $t \in T\}$ where $T$ is a subgroup of $G$ in which $G$ is the semidirect product of a normal subgroup $N$ and a subgroup $T$, and $\operatorname{Con}_{N}^{t}(f)$ is the conjugation of $t$ on $f$.

Proposition 3 (see [11]). Let $p$ and $q$ be distinct primes and let $G$ be a finite group of order $p^{2} q$. Then one of the following holds:

(i) $p>q$ and $G$ has a normal Sylow p-subgroup;

(ii) $p<q$ and $G$ has a normal Sylow $q$-subgroup;

(iii) $p=2, q=3, G \cong A_{4}$, and $G$ has a normal 2-subgroup.

Proposition 4 (see [9]). Let $G$ be a nonabelian group of order $p^{2} q$ where $p$ and $q$ are distinct primes. Then exactly one of the following holds:

(i) $G^{\prime} \cong \mathbb{Z}_{p}$ and $G^{a b} \cong \mathbb{Z}_{p q}$;

(ii) $G^{\prime} \cong \mathbb{Z}_{p^{2}}$ and $G^{a b} \cong \mathbb{Z}_{q}$;

(iii) $G^{\prime} \cong \mathbb{Z}_{p} \times \mathbb{Z}_{p}$ and $G^{a b} \cong \mathbb{Z}_{q}$;

(iv) $G^{\prime} \cong \mathbb{Z}_{q}$ and $G^{a b} \cong \mathbb{Z}_{p^{2}}$;

(v) $G^{\prime} \cong \mathbb{Z}_{q}$ and $G^{a b} \cong \mathbb{Z}_{p} \times \mathbb{Z}_{p}$;

(vi) $G^{\prime} \cong \mathbb{Z}_{2} \times \mathbb{Z}_{2}$.

Proposition 5 (see [12]). The factor group $G / G^{\prime}$ is abelian. If $K$ is a normal subgroup of $G$ such that $G / K$ is abelian, then $G^{\prime} \subseteq K$.

Proposition 6 (see [5]). Let $G \cong \mathbb{Z}_{m}$ and $H \cong \mathbb{Z}_{n}$ be cyclic groups that act trivially on each other. Then $G \otimes H \cong \mathbb{Z}_{(m, n)}$.

Proposition 7 (see [2]). Let $G$ be a finite group. Then

(i) $M(G)$ is a finite group whose elements have order dividing the order of $G$.

(ii) $M(G)=1$ if $G$ is cyclic.

Proposition 8 (see [2]). If the Sylow p-subgroups of $G$ are cyclic for all $p|| G \mid$, then $M(G)=1$.

Proposition 9 (see [2]). Let $N$ be a normal Hall subgroup of $G$ and $T$ a complement of $N$ in $G$. Then

$$
M(G) \cong M(T) \times M(N)^{T} .
$$

Proposition 10 (see [2]). If $G_{1}$ and $G_{2}$ are finite groups, then

$$
M\left(G_{1} \times G_{2}\right)=M\left(G_{1}\right) \times M\left(G_{2}\right) \times\left(G_{1} \otimes G_{2}\right) .
$$

Proposition 11 (see [6]). Let $G$ be a finite nonabelian group. If $G$ is a group of order $p^{2} q$, then

$$
M(G)= \begin{cases}1, & \text { if } G^{\prime}=\mathbb{Z}_{q}, G^{a b}=\mathbb{Z}_{p^{2}}, \\ \mathbb{Z}_{p}, & \text { if } G^{\prime}=\mathbb{Z}_{q}, G^{a b}=\mathbb{Z}_{p} \times \mathbb{Z}_{p}, \\ \mathbb{Z}_{2}, & \text { if } G^{a b}=\mathbb{Z}_{2} \times \mathbb{Z}_{2} .\end{cases}
$$

The following propositions are some of the basic results of the Schur multiplier of a pair deduced by Ellis [3], assuming only the existence of the natural exact sequence in (1) and the existence of a certain transfer homomorphism.

Proposition 12 (see [3]). Let $N=1$; then $M(G, N)=1$.

Proposition 13 (see [3]). Let $N=G$; then $M(G, G)=M(G)$.

Proposition 14 (see [3]). Suppose that $G$ is a finite group. Let the order of the normal subgroup $N$ be coprime to its index in $G$ and $T$ a complement of $N$ in $G$. Then $G \cong N \rtimes T$ and $M(G, N) \cong M(N)^{T}$.

\section{Main Result}

In the following two theorems, the Schur multipliers of pairs of groups of order $p^{2} q$ are stated and proved. We assume that the group is nonabelian.

Theorem 15. Let $G$ be a group of order $p^{2} q$ where $p$ and $q$ are distinct primes, and $p<q$. If $N \triangleleft G$, then the Schur multiplier of pairs of $G$

$$
M(G, N)= \begin{cases}1, & \text { if } G^{a b} \cong \mathbb{Z}_{p^{2}} \text { or } G^{a b} \cong \mathbb{Z}_{p} \times \mathbb{Z}_{p} \\ & \text { when } N=1 \text { or } \mathbb{Z}_{q}, \\ \mathbb{Z}_{p}, & \text { if } G^{a b} \cong \mathbb{Z}_{p} \times \mathbb{Z}_{p} \\ & \text { when } N=G, \mathbb{Z}_{p}, \mathbb{Z}_{p q}, \mathbb{Z}_{p} \times \mathbb{Z}_{p} \text { or } \mathbb{Z}_{p^{2}},\end{cases}
$$

where $G^{a b}=G / G^{\prime}$.

Proof. Let $G$ be a group of order $p^{2} q$ where $p$ and $q$ are distinct primes, and $p<q$. Since $p<q$, then by Proposition $3 G$ has a normal Sylow $q$-subgroup: call it $Q$. Moreover, $[G: Q]=p^{2}$ so $G / Q$ is abelian. Then by Proposition 5 , we have $G^{\prime} \subseteq Q$; that is, $G^{\prime}=\mathbb{Z}_{q}$. Thus, by Proposition $4, G^{a b} \cong \mathbb{Z}_{p^{2}}$ or $G^{a b} \cong$ $\mathbb{Z}_{p} \times \mathbb{Z}_{p}$.

Suppose $N \triangleleft G$; then the Schur multiplier of pairs of $G$ is computed below.

Case 1. If $G^{a b} \cong \mathbb{Z}_{p^{2}}$ then by Proposition $11, M(G)=1$.

Since $M(G)=1$, for all normal subgroups $N$ of $G$, $M(G, N) \leq M(G)=1$. 
Case 2. If $G^{a b} \cong \mathbb{Z}_{p} \times \mathbb{Z}_{p}$ then by Proposition $11, M(G)=\mathbb{Z}_{p}$.

(i) If $N=1$ then by Proposition 12, $M(G, N)=$ $M(G, 1)=1$.

(ii) If $N=G$ then by Proposition 13, $M(G, N)=$ $M(G, G)=M(G)$. By Proposition $11, M(G)=\mathbb{Z}_{p}$.

(iii) If $N=\mathbb{Z}_{q}$ then $G$ is the semidirect product of $\mathbb{Z}_{q}$ and $H$ in which $|N|$ and $[G: N]$ are coprimes, and $N$ is a normal Hall subgroup of $G$ (refer to Definition 1). Therefore by Proposition 14, $M(G, N)=M(N)^{H}=1$ since $M\left(\mathbb{Z}_{q}\right)=1$ (refer to Proposition 7). Note that, for this case, $G / N=G / G^{\prime} \neq \mathbb{Z}_{p^{2}}$; that is, $G / N \neq \mathbb{Z}_{p^{2}}$.

(iv) If $N=\mathbb{Z}_{p}$ then $G / N$ is nonabelian group of order $p q$. (If $G / N \cong \mathbb{Z}_{p q}$ then by Proposition $5, G^{\prime} \subseteq N$; that is, $\mathbb{Z}_{q} \subseteq \mathbb{Z}_{p}$ and this statement is a contradiction). Thus the exact sequence $M(G, N) \rightarrow M(G) \rightarrow$ $M(G / N)=1$ shows that $M(G, N) / \kappa \cong \mathbb{Z}_{p}$ where $\kappa$ is the kernel of homomorphism $M(G, N)$ to $M(G)$. Then $M(G, N)=\mathbb{Z}_{p}$.

(v) If $N=\mathbb{Z}_{p q}, \mathbb{Z}_{p^{2}}$ or $\mathbb{Z}_{p} \times \mathbb{Z}_{p}$ then by similar way as in (iv), $M(G, N)=\mathbb{Z}_{p}$.

Theorem 16. Let $G$ be a group of order $p^{2} q$ where $p$ and $q$ are distinct primes, and $p>q$. If $N \triangleleft G$, then the Schur multiplier of pairs of $G$

$$
\begin{aligned}
& M(G, N) \\
& = \begin{cases}1, & \text { if } G^{\prime} \cong \mathbb{Z}_{p} \text { or } G^{\prime} \cong \mathbb{Z}_{p^{2}}, \text { or } G^{\prime} \cong \mathbb{Z}_{p} \times \mathbb{Z}_{p} \\
\text { when } N=1 \text { or } \mathbb{Z}_{q}, \\
\mathbb{Z}_{p}, \quad \text { if } G^{\prime} \cong \mathbb{Z}_{p} \times \mathbb{Z}_{p} \\
\text { when } N=G, \mathbb{Z}_{p} \text { or } \mathbb{Z}_{p} \times \mathbb{Z}_{p},\end{cases}
\end{aligned}
$$

where $G^{a b}=G / G^{\prime}$.

Proof. Let $G$ be a group of order $p^{2} q$ where $p$ and $q$ are distinct primes, and $p>q$. Since $p>q, G$ has a normal Sylow $p$ subgroup, namely, $P$ (refer to Proposition 3 ). $[G: P]=q$ so $G / P$ is abelian. Hence, $G^{\prime} \subseteq P$ (refer to Proposition 5); that is, $G^{\prime} \cong \mathbb{Z}_{p} \times \mathbb{Z}_{p}, \mathbb{Z}_{p^{2}}$ or $\mathbb{Z}_{p}$. Suppose $N \triangleleft G$; then the Schur multiplier of pairs of $G$ is computed below.

(In this case $N=1, \mathbb{Z}_{q}, \mathbb{Z}_{p}, \mathbb{Z}_{p} \times \mathbb{Z}_{p}$ and $G$.)

Case 1. If $G^{\prime} \cong \mathbb{Z}_{p} \times \mathbb{Z}_{p}$ then $\left|G^{\prime}\right|=p^{2}$ and $\left[G: G^{\prime}\right]=q$ are coprimes. Then, by Definition $1, G^{\prime}$ is a normal Hall subgroup of $G$. Therefore by Proposition 9, $M(G)=M(T) \times M\left(G^{\prime}\right)^{T}$ where $T$ is a complement of $G^{\prime}$ and $T \cong \mathbb{Z}_{q}$. Thus, $M(G)=$ $M(T) \times M\left(\mathbb{Z}_{p} \times \mathbb{Z}_{p}\right)^{T} . M(T)=1$ (refer to Proposition 7). Hence, $M(G)=\mathbb{Z}_{p}$ (refer to Propositions 10, 6, and 7).

(i) If $N=1$ then $M(G, N)=M(G, 1)=1$ (refer to Proposition 12).

(ii) If $N=G$ then $M(G, N)=M(G, G)=M(G)$ (refer to Proposition 13). Then, $M(G)=\mathbb{Z}_{p}$. (iii) If $N=\mathbb{Z}_{q}$ then $N$ is a normal Hall subgroup of $G$ (refer to Definition 1) and $G$ is the semidirect product of $N$ and $H$ in which $H$ is a complement of $N$ in $G$. Therefore by Proposition 14, $M(G, N)=M(N)^{H}=1$ since $M\left(\mathbb{Z}_{q}\right)=1$ (refer to Proposition 7).

(iv) If $N=\mathbb{Z}_{p}$ then $G / N$ is nonabelian group of order $p q$. (If $G / N \cong \mathbb{Z}_{p q}$ then by Proposition $5, G^{\prime} \subseteq N$; that is, $\mathbb{Z}_{p} \times \mathbb{Z}_{p} \subseteq \mathbb{Z}_{p}$ and this statement is a contradiction). Thus the exact sequence $M(G, N) \rightarrow M(G) \rightarrow$ $M(G / N)=1$ shows that $M(G, N) / \kappa \cong \mathbb{Z}_{p}$ where $\kappa$ is the kernel of homomorphism $M(G, N)$ to $M(G)$. Then $M(G, N)=\mathbb{Z}_{p}$.

(v) If $N=\mathbb{Z}_{p} \times \mathbb{Z}_{p}$ then by similar way as in (iv), $M(G, N)=\mathbb{Z}_{p}$.

(vi) If $N=\mathbb{Z}_{p q}$ or $\mathbb{Z}_{p^{2}}$ then $G / N$ is abelian group and $G^{\prime} \cong \mathbb{Z}_{p} \times \mathbb{Z}_{p} \subseteq N \cong \mathbb{Z}_{p q}$ or $\mathbb{Z}_{p^{2}}$ but this statement is a contradiction. So $M(G, N)$ when $N=\mathbb{Z}_{p q}$ or $\mathbb{Z}_{p^{2}}$ are not considered.

Case 2. If $G^{\prime} \cong \mathbb{Z}_{p^{2}}$ then $G / G^{\prime} \cong G^{a b} \cong \mathbb{Z}_{q}$. Hence, all Sylow subgroups of $G$ are cyclic. Therefore, by Proposition 8 , $M(G)=1$. Thus, for all normal subgroups $N$ of $G, M(G, N) \leq$ $M(G)=1$.

Case 3. If $G^{\prime} \cong \mathbb{Z}_{p}$ then $M(G)=1$ since $M(G)=M\left(G^{\prime}\right) \times$ $M(K)$ where $K$ is a group of order $p q$. By Propositions 7 and $8, M\left(G^{\prime}\right)=1$ and $M(K)=1$. Thus, for all normal subgroups $N$ of $G, M(G, N) \leq M(G)=1$.

\section{Conclusion}

For a group $G$ of order $p^{2} q$ where $p$ and $q$ are prime numbers, $Q$ is the unique normal Sylow $q$-subgroups of $G$ if $p<q$, while $P$ is the unique normal Sylow $p$-subgroups of $G$ if $p>$ $q$. In this paper, we determined the Schur multiplier of pairs of groups of order $p^{2} q$. Our proofs show that $M(G, N)$ for groups of order $p^{2} q$ is either 1 or $\mathbb{Z}_{p}$.

\section{Conflict of Interests}

The authors declare that there is no conflict of interests regarding the publication of this paper.

\section{Acknowledgments}

The authors would like to thank Ministry of Education (MOE), Malaysia, and Research Management Centre Universiti Teknologi Malaysia (RMCUTM) for the financial support through the Research University Grant (RUG) Vote no. 04H13. The second author would also like to thank Ministry of Education (MOE), Malaysia, for her MyPhD Scholarship.

\section{References}

[1] I. Schur, "Über die Darstellung der endlichen gruppen durch gebrochen lineare substitutionen," Journal für die Reine und 
Angewandte Mathematik, vol. 1904, no. 127, pp. 20-50, 1904, 1904.

[2] G. Karpilovsky, The Schur Multiplier, Clarendon Press, Oxford, UK, 1987.

[3] G. Ellis, "The Schur multiplier of a pair of groups," Applied Categorical Structures, vol. 6, no. 3, pp. 355-371, 1998.

[4] R. Brown and J.-L. Loday, "Van Kampen theorems for diagrams of spaces," Topology, vol. 26, no. 3, pp. 311-335, 1987.

[5] M. Visscher, On the nonabelian tensor products of groups [Ph.D. dissertation], State University of New York at Binghamton, 1998.

[6] S. H. Jafari, P. Niroomand, and A. Erfanian, "The nonabelian tensor square and Schur multiplier of groups of order $p^{2} q, p q^{2}$ and $p^{2}$ qr," Algebra Colloquium, vol. 10, pp. 1083-1088, 2012.

[7] M. R. R. Moghaddam, A. R. Salemkar, and K. Chiti, "Some properties on the Schur multiplier of a pair of groups," Journal of Algebra, vol. 312, no. 1, pp. 1-8, 2007.

[8] S. Rashid, N. H. Sarmin, A. Erfanian, and N. M. Mohd Ali, "On the commutator subgroups of groups of order 8q," Journal of Computer Science \& Computational Mathematics, vol. 2, no. 2, pp. 5-7, 2012.

[9] S. Rashid, N. H. Sarmin, A. Erfanian, and N. M. Ali, "On the nonabelian tensor square and capability of groups of order $p^{2} q$," Archiv der Mathematik, vol. 97, no. 4, pp. 299-306, 2011.

[10] S. Rashid, N. H. Sarmin, A. Erfanian, N. M. Ali, and R. Zainal, "On the nonabelian tensor square and capability of groups of order 8q," Indagationes Mathematicae: New Series, vol. 24, no. 3, pp. 581-588, 2013.

[11] M. Quick, MT5824 Topics in Groups Lecture Notes, University of St. Andrews, 2004-2009.

[12] M. Hall, The Theory of Groups, Macmillan, New York, NY, USA, 1959. 


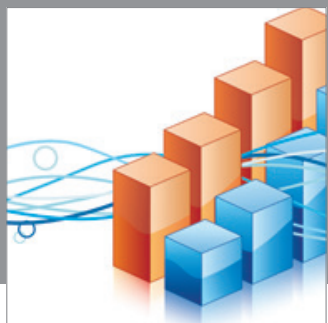

Advances in

Operations Research

mansans

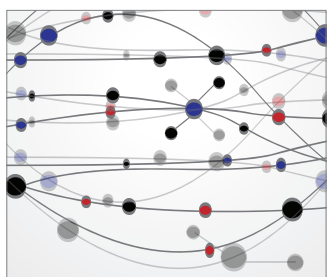

The Scientific World Journal
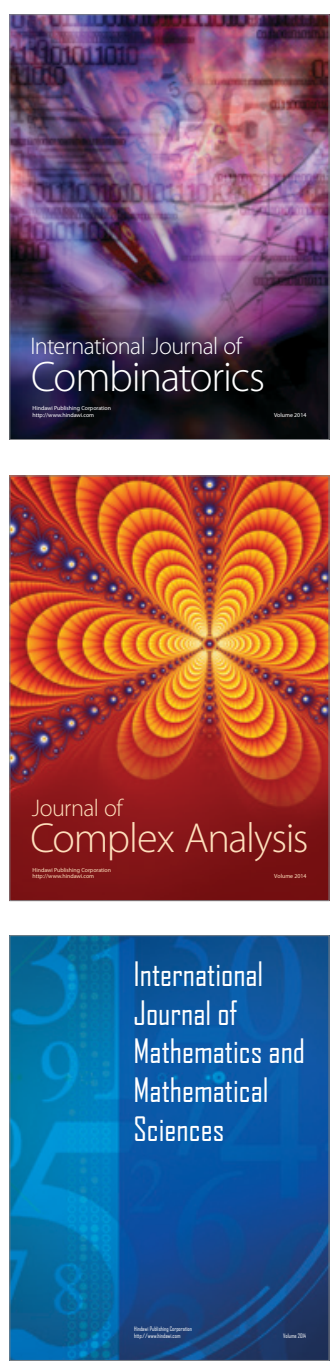
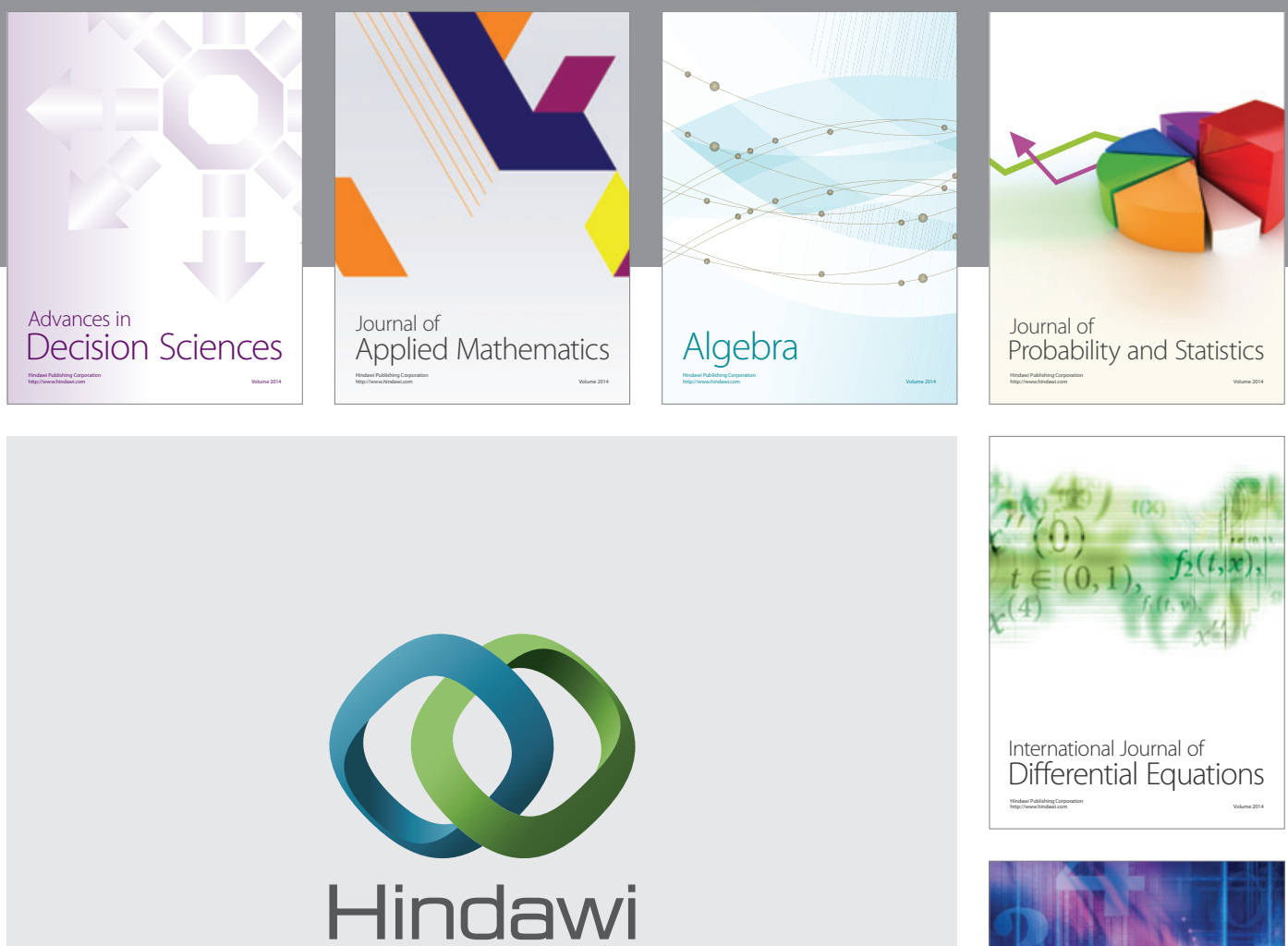

Submit your manuscripts at http://www.hindawi.com
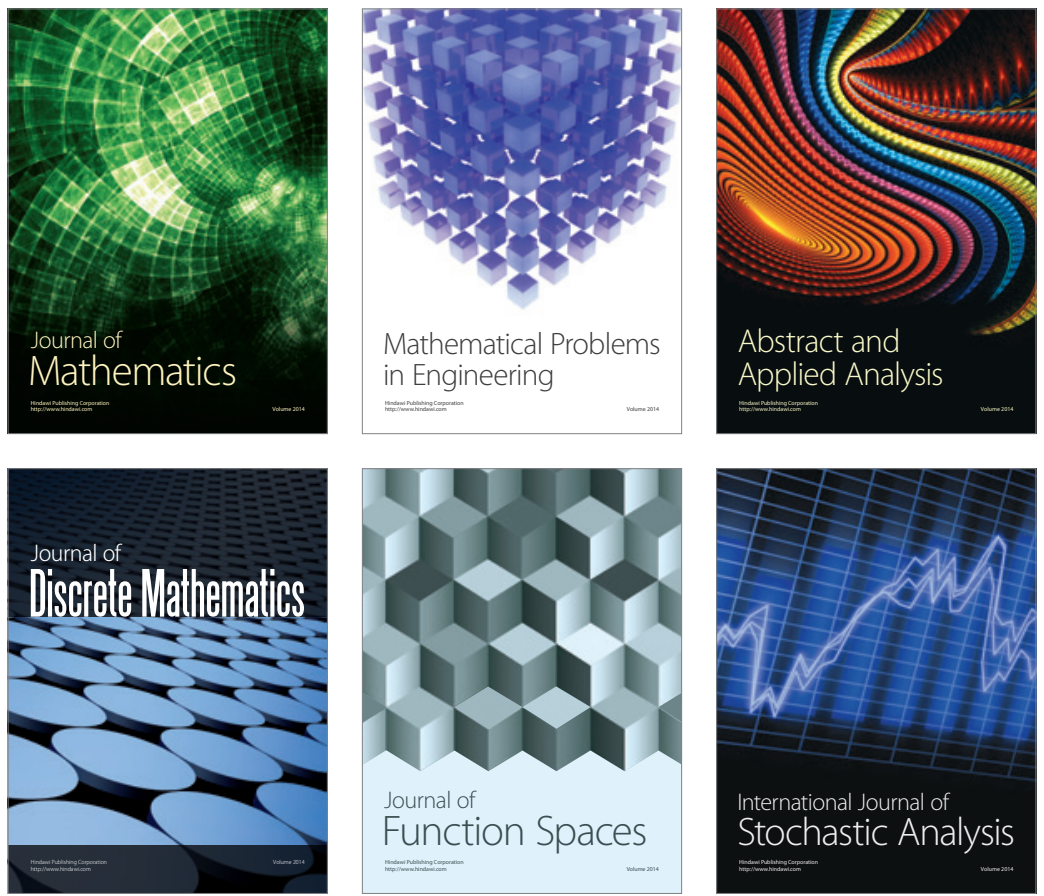

Journal of

Function Spaces

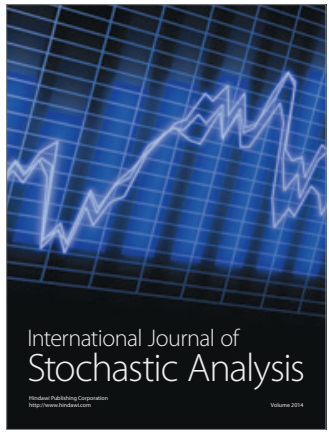

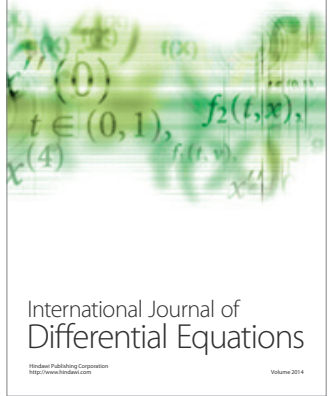
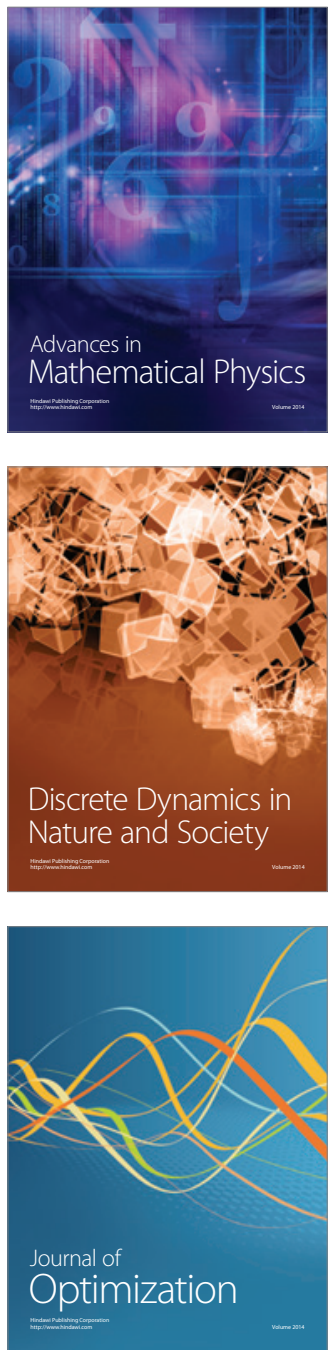